\title{
FIRST-ORDER MATHEMATICAL FUZZY LOGIC WITH HEDGES
}

\author{
Van-Hung Le \\ Faculty of Information Technology, \\ Hanoi University of Mining and Geology, Vietnam \\ levanhung@humg.edu.vn
}

\begin{abstract}
In this paper, we consider first-order mathematical fuzzy logic expanded by many hedges. This is based on the fact that, in the real world, many hedges can be used simultaneously, and some hedge modifies truth (or meaning of sentences) more than another hedge. Moreover, each hedge may or may not have a dual one. We expand two axiomatizations for propositional mathematical fuzzy logic with many hedges to the first-order level and prove a number of completeness results for the resulting logics. We also consider logics with many hedges based on $\Delta$-core fuzzy logics.
\end{abstract}

\section{KEYWORDS}

Mathematical Fuzzy Logic, First-Order Logic, Hedge, Strong Completeness, Standard Completeness

\section{INTRODUCTION}

Extending logical systems of mathematical fuzzy logic (MFL) with hedges is axiomatized by Hájek [1], Vychodil [2], Esteva et al. [3], among others. Hedges are called truthstressing or truth-depressing if they, respectively, strengthen or weaken the meaning of the applied proposition. Intuitively, on a chain of truth values, the truth function of a truth-depressing (resp., truth-stressing) hedge (connective) is a superdiagonal (resp., subdiagonal) non-decreasing function preserving 0 and 1 . In $[1,2,3]$, logical systems of MFL are extended by a truth-stressing hedge and/or a truth-depressing one.

However, in the real world, we often use many hedges, e.g., very, highly, rather, and slightly, at the same time to express different levels of emphasis. Furthermore, a hedge may or may not have a dual one, e.g., slightly (resp., rather) can be seen as a dual hedge of very (resp., highly). Therefore, in $[4,5]$, Le et al. propose two axiomatizations for propositional logical systems of MFL with many hedges. In the axiomatization in [5], each hedge does not have any dual one whereas in the axiomatization in [4], each hedge can have its own dual one. In $[5,6]$, logical systems with many hedges for representing and reasoning with linguistically-expressed human knowledge are also proposed.

David C. Wyld et al. (Eds) : DBDM, CICS, CSIP, AI\&FL, SCOM, CSE, CCNET-2016

pp. 85-96, 2016. (C) CS \& IT-CSCP 2016

DOI : $10.5121 /$ csit.2016.60509 
Moreover, since first-order logic is more powerful than propositional logic in terms of knowledge representation and reasoning. In this paper, we expand the axiomatizations in $[4,5]$ to the first-order level and prove a number of completeness results for the resulting logics w.r.t. the underlying logic. More concretely, we propose first-order fuzzy logics with many hedges based on a first-order core fuzzy logic for two cases: (i) each hedge does not have a dual one, and (ii) each hedge can have its own dual one. Then, we prove that the new first-order logics are a conservative expansion of the underlying first-order core fuzzy logic and gives a characterization of their strong completeness, especially, the strong standard completeness. We also discuss logics with many hedges based on $\Delta$-core fuzzy logics, which are extensions of core fuzzy logics by $\Delta$ connective.

The remainder of the paper is organized as follows. Section 2 gives an overview of notions and results of MFL used in this paper and the two axiomatizations for propositional MFL with many hedges proposed in $[4,5]$. Section 3 presents first-order MFL with many hedges and proves a number of completeness results. Section 4 discusses logics with many hedges based on $\Delta$-core fuzzy logics. Section 5 concludes the paper.

\section{PRELIMINARIES}

\subsection{Preliminaries on Mathematical Fuzzy Logic}

Let $\mathrm{L}$ be a propositional logic in a language $\mathcal{L}$, a set of connectives with finite arity. A truth constant $\bar{r}$ is a special formula whose truth value under every evaluation is $r$. Formulae are built from variables and truth constants using connectives in $\mathcal{L}$. Each evaluation $e$ of variables by truth values uniquely extends to an evaluation $e(\varphi)$ of all formulae $\varphi$ using truth functions of connectives. A formula $\varphi$ is called an 1-tautology if $e(\varphi)=1$ for all evaluations $e$. Several 1-tautology formulae are taken as axioms of the logic. A theory is a set of formulae. An evaluation $e$ is called a model of a theory $T$ if $e(\varphi)=1, \forall \varphi \in T$. A proof in $T$ is a sequence $\varphi_{1}, \ldots, \varphi_{n}$ of formulae whose each member is either an axiom of the logic or a member of $T$ or follows from some preceding members of the sequence using the deduction rule(s) of the logic. If $\varphi$ is the last member of a proof in $T, \varphi$ is called a provable formula, denoted $T \vdash_{L} \varphi$. If $T=\emptyset, \varphi$ is said to be provable in the logic [7, 8].

Most logical systems called core fuzzy logics are a finitary Rasiowa-implicative logic [8]. Every finitary Rasiowa-implicative logic $\mathrm{L}$ is algebraizable. Its equivalent algebraic semantics, a class of L-algebras, is a quasivariety. Each L-algebra A is endowed with a relation $\leq$ (called preorder) by setting, $\forall a, b \in A, a \leq b$ iff $a \Rightarrow b=1$, where $\Rightarrow$ is the truth function of the connective $\rightarrow$. If $\leq$ is a total order, $\mathbf{A}$ is called an L-chain. $\mathrm{L}$ is called a semilinear logic iff it is strongly complete w.r.t. the class of L-chains $[7,8]$. Core fuzzy logics are semilinear. They belong to a large class of systems which are axiomatic expansions of MTL (monoidal t-norm based logic). Other well-known examples of core

fuzzy logics are BL (basic logic), G (Gödel logic), Ł(Łukasiewicz logic), П (product logic) [7], SBL, NM, WNM, IMTL, and SMTL [8]. 
The $\Delta$ connective is the one whose truth function is given by: if $x=1, \Delta(x)=1$; otherwise, $\Delta(x)=0$. It is used to express the notion of full truth of a proposition which is not expressible in either MTL or other logics of left-continuous t-norms. A core fuzzy logic L expanded by this connective is called " $\mathrm{L}$ with $\Delta$ " and denoted by $L_{\Delta}$. It can pe axiomatized by adding the following axioms to the axiomatic system for the logic $\mathrm{L}[8]$ :

$$
\begin{array}{ll}
(\Delta 1) & \Delta \varphi \vee \neg \Delta \varphi \\
(\Delta 2) & \Delta(\varphi \vee \psi) \rightarrow \Delta \varphi \vee \Delta \psi \\
(\Delta 3) & \Delta \varphi \rightarrow \varphi \\
(\Delta 4) & \Delta \varphi \rightarrow \Delta \Delta \varphi \\
(\Delta 5) & \Delta(\varphi \rightarrow \psi) \rightarrow(\Delta \varphi \rightarrow \Delta \psi)
\end{array}
$$

and the rule of $\Delta$-necessitation: from $\varphi$ infer $\Delta \varphi$.

Besides the notion of full truth, the ordering of truth values is also internalized in $L_{\Delta}$, as $\Delta(x \rightarrow y)=1$ iff $x \leq y$. Moreover, the connective $\Delta$ makes it possible to interpret classical logic in $L_{\Delta}$ by prefixing each propositional variable by $\Delta$. Extensions of all core fuzzy logics by $\Delta$ are called $\Delta$-core fuzzy logics, e.g., MTL .

Definition 1 ( $\mathbb{K C}, \mathbf{F S} \mathbb{K C}, \mathbf{S K C})[8]$ Let $L$ be a core fuzzy logic and $\mathbb{K}$ a class of $L$ chains. It is said that $L$ has the (finitely) strong $\mathbb{K}$-completeness, ( $F$ ) S $\mathbb{K} C$ for short, if for every (finite) set of formulae $T$ and every formula $\varphi$, it holds that $T \vdash_{L} \varphi$ iff $e(\varphi)=1$ for every L-algebra $\mathbf{A} \in \mathbb{K}$ and each $\mathbf{A}$-model e of $T$. It is said that $L$ has the $\mathbb{K}$-completeness, $\mathbb{K} C$ for short, when the equivalence is true for $T=\emptyset$.

Clearly, the SKC implies the FSKC, and the FSIKC implies the $\mathbb{K C}$. When $\mathbb{K}$ is the class of all chains whose support is the unit interval $[0,1]$ with the usual ordering, the (F)SKC can be called the (finite) strong standard completeness, (F)SSC for short.

\subsection{An Axiomatization for Many Hedges}

A hedge may modify truth more than another [9, 10, 11]. For example, slightly (resp., very) modifies truth more than rather (resp., highly) since slightly true < rather true < true (resp., true $<$ highly true $<$ very true). To ease the presentation, let $s_{0}, d_{0}$ denote the identity connective, i.e., for all formula $\varphi, \varphi \equiv s_{0} \varphi \equiv d_{0} \varphi$, and their truth functions are the identity.

Definition 2 [5] Let $L$ be a core fuzzy logic. A logic $L_{s, d}^{p, q}$, where $p, q$ are positive integers, is an expansion of $L$ with new unary connectives $s_{1}, \ldots, s_{p}$ (for truth-stressers) and $d_{1}, \ldots, d_{q}$ (for truth-depressers) by the following additional axioms, for $i=1, \ldots, p$ and $j=1, \ldots, q$ :

$$
\begin{array}{cl}
\left(S_{i}\right) & s_{i} \varphi \rightarrow s_{i-1} \varphi \\
\left(S_{p+1}\right) & s_{p} \overline{1} \\
\left(D_{j}\right) & d_{j-1} \varphi \rightarrow d_{j} \varphi \\
\left(D_{q+1}\right) & \neg d_{q} \overline{0}
\end{array}
$$


and the following additional deduction rule:

$$
\left(D R^{h}\right) \text { from }(\varphi \rightarrow \psi) \vee \chi \text { infer }(h \varphi \rightarrow h \psi) \vee \chi, \text { for each } h \in\left\{s_{1}, \ldots, s_{p}, d_{1}, \ldots, d_{q}\right\} .
$$

Axiom $\left(S_{i}\right)$ (resp., axiom $\left(D_{j}\right)$ ) expresses that $s_{i}$ (resp., $d_{j}$ ) modifies truth more than $s_{i-1}$ (resp., $d_{j-1}$ ), for $i=2, \ldots, p$ (resp., $j=2, \ldots, q$ ).

It is shown that $L_{s, d}^{p, q}$ is a finitary Rasiowa-implicative logic [5], and its equivalent algebraic semantics is the class of $L_{s, d^{-}}^{p, q}$-algebras.

Definition 3 [5] An algebra $\boldsymbol{A}=\left\langle A, *, \Rightarrow, \cap, \cup, 0,1, s_{1}, \ldots, s_{p}, d_{1}, \ldots, d_{q}\right\rangle$ of type $\langle 2,2,2,2,0,0,1, \ldots, 1\rangle$ is an $L_{s, d}^{p, q}$-algebra if it is an L-algebra expanded by unary operators $s_{i}, d_{j}: A \rightarrow A$ that satisfy, for all $x, y, z \in A, i=\overline{1, p}$ and $j=\overline{1, q}$,

$$
\begin{array}{r}
s_{i}(x) \leq s_{i-1}(x) \\
s_{p}(1)=1 \\
d_{j}(x) \geq d_{j-1}(x) \\
d_{q}(0)=0 \\
\text { if }(x \Rightarrow y) \cup z=1 \text { then }\left(s_{i}^{\bullet}(x) \Rightarrow s_{i}^{\bullet}(y)\right) \cup z=1 \\
\text { if }(x \Rightarrow y) \cup z=1 \text { then }\left(d_{j}^{\bullet}(x) \Rightarrow d_{j}^{\bullet}(y)\right) \cup z=1
\end{array}
$$

In [5], it is proved that for all $i=\overline{1, p}, s_{i}$ is subdiagonal (i.e., $\forall x, s_{i}(x) \leq x$ ), and preserves 0 and 1 ; for all $j=\overline{1, q}, d_{j}$ is superdiagonal (i.e., $\forall x, d_{j}(x) \geq x$ ), and preserves 0 and 1 . Moreover, in a chain of truth values, they are all non-decreasing.

Theorem 1 [5] Let L be a core fuzzy logic, $\mathbb{K}$ a class of L-chains, and $\mathbb{K}_{s, d}^{p, q}$ the class of the $L_{s, d^{p}}^{p, q}$ chains whose $s_{1}, \ldots, s_{p}, d_{1}, \ldots, d_{q}$-free reducts are in $\mathbb{K}$. Then: (i) $L_{s, d}^{p, q}$ is a conservative expansion of $L$; (ii) $L_{s, d}^{p, q}$ is strongly complete w.r.t. the class of all $L_{s, d}^{p, q}$-chains, i.e., $L_{s, d}^{p, q}$ is semilinear; (iii) L has the FSSC, FSK C, SSC, and $S \mathbb{K} C$ iff $L_{s, d}^{p, q}$ has the FSSC, FSK $C$, $S S C$, and $S \mathbb{K} C$, respectively.

\subsection{Mathematical Fuzzy logic with Many Dual Hedges}

It can be observed that each hedge can have a dual one, e.g., slightly and rather can be seen as a dual hedge of very and highly, respectively. Thus, there might be axioms expressing dual relations of hedges in addition to axioms expressing their comparative truth modification strength.

Definition 4 [4] Let $L$ be a core fuzzy logic. A logic $L_{s, d}^{2 n}$, where $n$ is a positive integer, is an expansion of $L$ with new unary connectives $s_{1}, \ldots, s_{n}$ (for truth-stressers) and $d_{1}, \ldots, d_{n}$ (for truth-depressers) by the following additional axioms, for $i=1, \ldots, n$ : 


$$
\begin{array}{cl}
\left(S_{i}^{d h}\right) & s_{i} \varphi \rightarrow s_{i-1} \varphi \\
\left(S_{n+1}^{d h}\right) & s_{n} \overline{1} \\
\left(D_{i}^{d h}\right) & d_{i-1} \varphi \rightarrow d_{i} \varphi \\
\left(S D_{i}^{d h}\right) & d_{i} \varphi \rightarrow \neg s_{i} \neg \varphi
\end{array}
$$

and the following additional deduction rule:

$$
\left(D R^{d h}\right) \text { from }(\varphi \rightarrow \psi) \vee \chi \text { infer }(h \varphi \rightarrow h \psi) \vee \chi, \text { for } h \in\left\{s_{1}, \ldots, s_{n}, d_{1}, \ldots, d_{n}\right\} .
$$

The logic $\mathrm{L}_{s, d}^{2 n}$ is $\mathrm{L}$ expanded by $2 n$ hedges, where hedges are divided into pairs of dual ones. Axiom $\left(S D_{i}\right)$ expresses the dual relation between hedges $s_{i}$ and $d_{i}$ and coincides with Axiom (ST2) in Vychodil's axiomatization. For the case of very, slightly, and $\varphi=$ young, it means "slightly young implies not very old". It can be proved that $\left(S D_{i}^{d h}\right)$ is equivalent to $s_{i} \varphi \rightarrow \neg d_{i} \neg \varphi$ which implies "very young implies not slightly old" as well.

$L_{s, d}^{2 n}$ is also a finitary Rasiowa-implicative logic, and its equivalent algebraic semantics is the class of $L_{s, d^{-}}^{2 n}$ algebras.

Definition 5 [4] An algebra $\boldsymbol{A}=\left\langle A, *, \Rightarrow, \cap, \cup, 0,1, s_{1}, \ldots, s_{n}, d_{1}, \ldots, d_{n}\right\rangle$ of type $\langle 2,2,2,2,0,0,1, \ldots, 1\rangle$ is an $L_{s, d^{-}}^{2 n}$ algebra if it is an L-algebra expanded by unary operators $s_{i}, d_{i}: A \rightarrow A$ that satisfy, for all $x, y, z \in A$ and $i=1, \ldots, n$,

$$
\begin{array}{r}
s_{i}(x) \leq s_{i-1}(x) \\
d_{i}(x) \geq d_{i-1}(x) \\
s_{n}(1)=1 \\
d_{i}(x) \leq-s_{i}(-x) \\
\text { if }(x \Rightarrow y) \cup z=1 \text { then }\left(s_{i}(x) \Rightarrow s_{i}(y)\right) \cup z=1 \\
\text { if }(x \Rightarrow y) \cup z=1 \text { then }\left(d_{i}(x) \Rightarrow d_{i}(y)\right) \cup z=1
\end{array}
$$

Theorem 2 [4] Let $L$ be a core fuzzy logic, $\mathbb{K}$ a class of L-chains, and $\mathbb{K}_{s, d}^{2 n}$ the class of the $L_{s, d}^{2 n}$-chains whose $s_{1}, \ldots, s_{n}, d_{1}, \ldots, d_{n}$-free reducts are in $\mathbb{K}$. Then: (i) $L_{s, d}^{2 n}$ is a conservative expansion of $L$; (ii) $L_{s, d}^{2 n}$ is strongly complete w.r.t. the class of all $L_{s, d}^{2 n}$-chains, i.e., $L_{s, d}^{2 n}$ is semilinear; (iii) L has the FSSC, FSK C, SSC, and $S \mathbb{K} C$ iff $L_{s, d}^{2 n}$ has the the FSSC, FSK $C$, $S S C$, and $S \mathbb{K} C$, respectively.

It can be seen that in a case when there is one truth-stressing (resp., truth-depressing) hedge without a dual one, we just add the axioms expressing its relations to the existing truth-stressing (resp., truth-depressing) hedges according to their comparative truth modification strength. 


\section{FIRST-ORDER MATHEMATICAL FUZZY LOGIC WITH HEDGES}

We first recall the representation of first-order formalisms for core fuzzy logics [8]. Given a propositional core fuzzy logic L, the language $\mathcal{P} \mathcal{L}$ of the first-order core fuzzy logic $\mathrm{L} \forall$ is built from the propositional language $\mathcal{L}$ of $\mathrm{L}$ by extending it with a non-empty set of predicate symbols Pred, a set of function symbols Func (disjoint with Pred), a set of object variables Var, and two quantifiers $\exists$ and $\forall$. The set of terms Term is the minimum set containing the elements of $V a r$ and closed under the functions. The atomic formulae are in the form of $P\left(t^{1}, \ldots, t^{n}\right)$, where $P \in$ Pred and $t^{1}, \ldots, t^{n} \in$ Term. The set of all formulae is obtained by closing the set of atomic formulae under combination by propositional connectives and quantification, i.e., if $u$ is a formula and $x$ is a variable, then $(\forall x) u$ and $(\exists x) u$ are formulae.

In first-order core fuzzy logics, it is usual to restrict the semantics to chains only. Given an L-chain $\mathbf{A}$, an $\mathbf{A}$-structure is $\mathbf{M}=\left\langle M,\left(P_{\mathbf{M}}\right)_{P \in P r e d},\left(f_{\mathbf{M}}\right)_{f \in F u n c}\right\rangle$, where $M$ is a non-empty domain; for each $n$-ary predicate symbol $P \in$ Pred, $P_{M}$ is an $n$-ary fuzzy relation on $M$, i.e., a function $M^{n} \rightarrow A$ (identified with an element of $A$ if $n=0$ ); for each $n$-ary function symbol $f \in$ Func, $f_{\mathrm{M}}$ is a function $M^{n} \rightarrow M$ (identified with an element of $M$ if $n=0$ ). An evaluation $v$ of variables is a mapping $v: \operatorname{Var} \rightarrow M$. Let $x$ be a variable and $a \in M$. Then let $v[x \rightarrow a]$ denote the evaluation such that $v[x \rightarrow a](x)=a$ and $v[x \rightarrow a](y)=y$ for each variable $y$ different from $x$. The values of terms and the truth values of formulae are defined inductively as follows:

$$
\begin{aligned}
\|x\|_{\mathbf{M}, v}^{\mathbf{A}}=v(x) & \\
\left\|f\left(t^{1}, \ldots, t^{n}\right)\right\|_{\mathbf{M}, v}^{\mathbf{A}}=f_{\mathbf{M}}\left(\left\|t^{1}\right\|_{\mathbf{M}, v}^{\mathbf{A}}, \ldots,\left\|t^{n}\right\|_{\mathbf{M}, v}^{\mathbf{A}}\right) & \text { for } f \in \text { Func } \\
\left\|P\left(t^{1}, \ldots, t^{n}\right)\right\|_{\mathbf{M}, v}^{\mathbf{A}}=P_{\mathbf{M}}\left(\left\|t^{1}\right\|_{\mathbf{M}, v}^{\mathbf{A}}, \ldots,\left\|t^{n}\right\|_{\mathbf{M}, v}^{\mathbf{A}}\right) & \text { for } P \in \text { Pred } \\
\left\|c\left(\varphi_{1}, \ldots, \varphi_{n}\right)\right\|_{\mathbf{M}, v}^{\mathbf{A}}=c_{\mathbf{A}}\left(\left\|\varphi_{1}\right\|_{\mathbf{M}, v}^{\mathbf{A}}, \ldots,\left\|\varphi_{n}\right\|_{\mathbf{M}, v}^{\mathbf{A}}\right) & \text { for } c \in \mathcal{L} \\
\|(\forall x) \varphi\|_{\mathbf{M}, v}^{\mathbf{A}}=\inf \left\{\|\varphi\|_{\mathbf{M}, v[x \rightarrow a]}^{\mathbf{A}} \mid a \in M\right\} & \\
\|(\exists x) \varphi\|_{\mathbf{M}, v}^{\mathbf{A}}=\sup \left\{\|\varphi\|_{\mathbf{M}, v[x \rightarrow a]}^{\mathbf{A}} \mid a \in M\right\} &
\end{aligned}
$$

if the infimum or supremum does not exist, the truth value of the quantified formula is undefined. The A-structure $\mathbf{M}$ is safe if $\|\varphi\|_{\mathbf{M}, v}^{\mathbf{A}}$ is defined for each formula $\varphi$ and each evaluation $v$. Then the truth value of $\varphi$ in a safe A-structure $\mathbf{M}$ is just:

$$
\|\varphi\|_{\mathbf{M}}^{\mathbf{A}}=\inf \left\{\|\varphi\|_{\mathbf{M}, v}^{\mathbf{A}} \mid v: \operatorname{Var} \rightarrow M\right\}
$$

For a safe $\mathbf{A}$-structure $\mathbf{M}$, if $\|\varphi\|_{\mathbf{M}}^{\mathbf{A}}=1$, the pair $\langle\mathbf{A}, \mathbf{M}\rangle$ is said to be a model of $\varphi$, denoted $\langle\mathbf{A}, \mathbf{M}\rangle \models \varphi$. A pair $\langle\mathbf{A}, \mathbf{M}\rangle$ is called a model of a theory (a set of sentences) $T$ if $\langle\mathbf{A}, \mathbf{M}\rangle \models \varphi$ for each $\varphi \in T$.

The axioms for $\mathrm{L} \forall$ are obtained from those of $\mathrm{L}$ by substituting formulae of $\mathcal{P} \mathcal{L}$ for propositional variables and adding the following axioms for quantifiers: 


$$
\begin{array}{cl}
(\forall x) \varphi(x) \rightarrow \varphi(t), & \text { where the term } t \text { is substitutable for } x \text { in } \varphi \\
\varphi(t) \rightarrow(\exists x) \varphi(x), & \text { where } t \text { is substitutable for } x \text { in } \varphi \\
(\forall x)(\psi \rightarrow \varphi) \rightarrow(\psi \rightarrow(\forall x) \varphi), & \text { where } x \text { is not free in } \psi \\
(\forall x)(\varphi \rightarrow \psi) \rightarrow((\exists x) \varphi \rightarrow \psi), & \text { where } x \text { is not free in } \psi \\
(\forall x)(\psi \vee \varphi) \rightarrow(\psi \vee(\forall x) \varphi), & \text { where } x \text { is not free in } \psi
\end{array}
$$

The deduction rules of $\mathrm{L} \forall$ are modus ponens and generalization:

$$
(\text { Gen }) \quad \text { From } \varphi \text { infer }(\forall x) \varphi
$$

The notions of proof and provability are defined in the usual way. The formula $\varphi$ is provable in $L \forall$ from a theory $T$ is denoted by $T \vdash_{L \forall} \varphi$.

The syntax and semantics of first-order core fuzzy logics are bounded together by the following completeness theorem:

Theorem 3 [12] For any first-order core fuzzy logic $L \forall$ with a predicate language $\mathcal{P} \mathcal{L}$, any $\mathcal{P} \mathcal{L}$-theory $T$, and any $\mathcal{P} \mathcal{L}$-formula $\varphi$, the following are equivalent:

- $T \vdash_{L \forall} \varphi$

- $\langle\mathbf{A}, \mathbf{M}\rangle \models \varphi$ for each model $\langle\mathbf{A}, \mathbf{M}\rangle$ of $T$ with $\mathbf{A}$ being a countable L-chain.

The notions $\mathrm{S} \mathbb{K C}, \mathrm{FSKC}$, and $\mathbb{K C}$ are defined similarly to the propositional case as follows:

Definition 6 [8] Let L $\forall$ be a core first-order fuzzy logic and $\mathbb{K}$ a class of L-chains. L $\forall$ has the (finite) strong $\mathbb{K}$-completeness property, $(F) S \mathbb{K} C$, if for every (finite) set of formulae $T$ and every formula $\varphi$, it holds that $T \vdash_{L \forall} \varphi$ iff $\langle\mathbf{A}, \mathbf{M}\rangle \models \varphi$ for every L-algebra $\mathbf{A}$ of $\mathbb{K}$ and each model $\langle\mathbf{A}, \mathbf{M}\rangle$ of $T$. L has the $\mathbb{K}$-completeness property, $\mathbb{K} C$, when the equivalence is true for $T=\emptyset$.

When $\mathbb{K}$ is the class of all chains whose support is the unit interval $[0,1]$ with the usual ordering, the (F)SKC can be called the (finite) strong standard completeness, (F)SSC.

Theorem 3 states that every first-order core fuzzy logic enjoys the SKC, where $\mathbb{K}$ is the class of all countable chains.

First-order fuzzy logics with many hedges in which each hedge does not have any dual one can be defined as follows.

Definition 7 Given a first-order core fuzzy logic $L \forall$, let $L_{s, d}^{p, q} \forall$ be the expansion of $L \forall$ with new unary connectives $s_{1}, \ldots, s_{p}$ (for truth-stressers) and $d_{1}, \ldots, d_{q}$ (for truth-depressers), axioms $\left(S_{i}\right),\left(S_{p+1}\right),\left(D_{j}\right),\left(D_{q+1}\right)$, and the deduction rule $\left(D R^{h}\right)$.

$\mathrm{L}_{s, d}^{p, q} \forall$ can be obtained by expanding $\mathrm{L}_{s, d}^{p, q}$ to the first-order level in the similar way as above. 
Theorem 4 The logic $L_{s, d}^{p, q} \forall$ is a conservative expansion of the logic $L \forall$, i.e., for every set $T \cup \varphi$ of $\mathcal{P} \mathcal{L}$-formulae, $T \vdash_{L_{s, d}^{p, q} \forall} \varphi$ iff $T \vdash_{L \forall} \varphi$, where $\mathcal{P} \mathcal{L}$ is the language of $L \forall$.

Proof. It is trivial that if $T \vdash_{L \forall} \varphi$ then $T \vdash_{L_{s, d}^{p, q} \forall} \varphi$. Now assume that $T \nvdash_{L \forall} \varphi$. Then, there exists an L-chain $\mathbf{A}$ and a model $\langle\mathbf{A}, \mathbf{M}\rangle$ of $T$ such that $\langle\mathbf{A}, \mathbf{M}\rangle \not \models \varphi$. A can be expanded to an $\mathrm{L}_{s, d^{-}}^{p, q}$ chain $\mathbf{A}^{\prime}$ by defining $s_{i}^{\bullet}(1)=1$ and $s_{i}^{\bullet}(a)=0$ for every $a \in A \backslash\{1\}(i=\overline{1, p})$, and $d_{j}^{\bullet}(0)=0$ and $d_{j}^{\bullet}(a)=1$ for every $a \in A \backslash\{0\}(j=\overline{1, q})$. It can be easily verified that the defined functions satisfy Conditions (1)-(6). Thus, in the expanded language of $\mathcal{P} \mathcal{L}$, $\left\langle\mathbf{A}^{\prime}, \mathbf{M}\right\rangle$ is still safe and is a model of $T$, but $\left\langle\mathbf{A}^{\prime}, \mathbf{M}\right\rangle \not \models \varphi$, i.e., $T \nvdash_{L_{s, d}^{p, q} \forall} \varphi$.

Let A, B be two algebras of the same type with (defined) lattice operations sup, inf. It is said that an embedding $f: \mathbf{A} \rightarrow \mathbf{B}$ is a $\sigma$-embedding if $f(\sup C)=\sup f[C]$ (whenever $\sup C$ exists) and $f(\inf D)=\inf f[D]$ (whenever inf $D$ exists) for each countable $C, D \subseteq A$, i.e., $f$ preserves existing suprema and infima. As shown in [12], a usual way to prove $\mathrm{SKC}$ is to show that every non-trivial countable L-chain can be $\sigma$-embedded into some chain of $\mathbb{K}$. In this case, it is said that $\mathrm{L}$ has the $\mathbb{K}$ - $\sigma$-embedding property. As proved in [12], this is a sufficient, but in general not necessary, condition for the SKC. This method has been used to prove strong real completeness for first-order versions of a number of important core fuzzy logics, e.g., MTL, SMTL, IMTL, G, NM, and WNM.

The SSC of the logic $\mathrm{L}_{\mathrm{s} d}^{p, q} \forall$ is characterized in the following theorem.

Theorem 5 (Strong standard completeness) Let L be a core fuzzy logic, $\mathbb{K}$ the class of all standard $L$-chains, and $\mathbb{K}_{s, d}^{p, q}$ the class of all standard $L_{s, d}^{p, q}$-chains whose $s_{1}, \ldots, s_{p}, d_{1}, \ldots, d_{q^{-}}$ free reducts are in $\mathbb{K}$. Then:

(i) If $L$ has the $\mathbb{K}$ - $\sigma$-embedding property, then $L_{s, d}^{p, q} \forall$ has the $S \mathbb{K}_{s, d}^{p, q} C$, i.e., SSC.

(ii) If $L$ does not have $\mathbb{K}$-embedding property, then $L_{s, d}^{p, q} \forall$ does not have the $S \mathbb{K}_{s, d}^{p, q} C$.

Proof. For (i), it suffices to prove that any non-trivial countable $\mathrm{L}_{s, d}^{p, q}$-chain A can be $\sigma$-embedded into some standard chain of $\mathbb{K}_{s, d}^{p, q}$. Since $\mathrm{L}$ has the $\mathbb{K}$ - $\sigma$-embedding property, the $s_{1}, \ldots, s_{p}, d_{1}, \ldots, d_{q}$-free reduct of $\mathbf{A}$ can be $\sigma$-embedded into a standard L-chain $\mathbf{B}=$ $\langle[0,1], *, \Rightarrow, \cap, \cup, 0,1\rangle$ by a mapping $f$ preserving existing suprema and infima. Since $\mathbf{A}$ is countable, for each $1 \leq k \leq p$, we may arrange all points $\left\{\left\langle f(x), f\left(s_{k}(x)\right)\right\rangle \mid x \in A\right\}$ into a sequence $\left\{\left\langle f\left(x_{i}\right), f\left(s_{k}\left(x_{i}\right)\right)\right\rangle \mid x_{i} \in A, i=1,2, \ldots\right\}$, where $0=x_{1}<x_{2}<\ldots$ and $\lim _{i \rightarrow \infty} x_{i}=1$. Since $f$ preserves the order relation, we have $0=f\left(x_{1}\right)<f\left(x_{2}\right)<\ldots$ and $\lim _{i \rightarrow \infty} f\left(x_{i}\right)=1$. Let $s_{k}^{\prime}:[0,1] \rightarrow[0,1]$ be the piecewise linear function connecting neighbored points from $\left\{\left\langle f\left(x_{i}\right), f\left(s_{k}\left(x_{i}\right)\right)\right\rangle\right\}$, i.e., $s_{k}^{\prime}$ is continuous and $s_{k}^{\prime}(f(x))=f\left(s_{k}(x)\right)$, and $s_{k}^{\prime}(1)=1$. Similarly, for each $1 \leq l \leq q$, let $d_{l}^{\prime}$ be the piecewise linear function connecting neighbored points from $\left\{\left\langle f\left(x_{i}\right), f\left(d_{l}\left(x_{i}\right)\right)\right\rangle\right\}$, i.e., $d_{l}^{\prime}$ is continuous $d_{l}^{\prime}(f(x))=f\left(d_{l}(x)\right)$, and $d_{l}^{\prime}(1)=1$. We will show that all $s_{k}^{\prime}$ and $d_{l}^{\prime}$ also satisfy Conditions (1)-(6). Since $s_{k}$ and $d_{l}$ preserve 0, clearly, $s_{k}^{\prime}$ and $d_{l}^{\prime}$ also preserve 0 . By (1), we have $s_{k}(x) \leq s_{k-1}(x)$, for all $1 \leq k \leq p$ (note that $\left.s_{0}(x)=x\right)$. Thus, $s_{k}^{\prime}\left(f\left(x_{i}\right)\right)=f\left(s_{k}\left(x_{i}\right)\right) \leq f\left(s_{k-1}\left(x_{i}\right)\right)=s_{k-1}^{\prime}\left(f\left(x_{i}\right)\right)$ for all $i$. Since $s_{k}^{\prime}, s_{k-1}^{\prime}$ are the piecewise linear functions connecting the corresponding points, we have $s_{k}^{\prime}(x) \leq s_{k-1}^{\prime}(x)$ for all $x \in[0,1]$. Similarly, we have $d_{l}^{\prime}(x) \geq d_{l-1}^{\prime}(x)$ for all $x \in[0,1]$. It remains to show that all $s_{k}^{\prime}$ and $d_{l}^{\prime}$ are non-decreasing. For all $i \geq 1$, since $f$ and $s_{k}$ are non-decreasing, we have $f\left(x_{i}\right)<f\left(x_{i+1}\right)$ and $s_{k}^{\prime}\left(f\left(x_{i}\right)\right)=$ 
$f\left(s_{k}\left(x_{i}\right)\right) \leq f\left(s_{k}\left(x_{i+1}\right)\right)=s_{k}^{\prime}\left(f\left(x_{i+1}\right)\right)$. Also, since $s_{k}^{\prime}$ linearly connects neighbored points from $\left\{\left\langle f\left(x_{i}\right), s_{k}^{\prime}\left(f\left(x_{i}\right)\right)\right\rangle \mid i=1,2, \ldots\right\}, s_{k}^{\prime}$ is non-decreasing on [0,1]. Similarly, $d_{l}^{\prime}$ is nondecreasing on $[0,1]$. Hence, $\mathrm{B}$ expanded by all $s_{k}^{\prime}$ and $d_{l}^{\prime}$ is a standard $\mathrm{L}_{s, d^{-}}^{p, q}$ chain into which $\mathbf{A}$ is $\sigma$-embedded.

For (ii), since L does not have the $\mathbb{K}$-embedding property, L $\forall$ does not enjoy the SKC. Moreover, since $\mathrm{L}_{s, d}^{p, q} \forall$ is a conservative expansion of $\mathrm{L} \forall, \mathrm{L}_{s, d}^{p, q} \forall$ does not have the $\mathrm{SK}_{s, d}^{p, q} \mathrm{C}$ either.

It can be seen from the proof that if we only know L has the K-embedding property, we cannot conclude anything, in general, about the SSC of $\mathrm{L}_{s, d}^{p, q} \forall$.

Theorem 5 can be generalized to arbitrary classes of L-chains and their $s_{1}, \ldots, s_{p}, d_{1}, \ldots, d_{q^{-}}$ expansions, which can be proved in an analogous way, as follows.

Corollary 1 (Strong $\mathbb{K}$-completeness) Let $L$ be a core fuzzy logic, $\mathbb{K}$ a class of $L$ chains, and $\mathbb{K}_{s, d}^{p, q}$ the class of the $L_{s, d}^{p, q}$-chains whose $s_{1}, \ldots, s_{p}, d_{1}, \ldots, d_{q}$-free reducts are in $\mathbb{K}$. Then:

(i) If $L$ has the $\mathbb{K}$ - $\sigma$-embedding property, then $L_{s, d}^{p, q} \forall$ has the $S \mathbb{K}_{s, d}^{p, q} C$.

(ii) If $L$ does not have $\mathbb{K}$-embedding property, then $L_{s, d}^{p, q} \forall$ does not have the $S \mathbb{K}_{s, d}^{p, q} C$.

Similarly, first-order fuzzy logics with many hedges in which each hedge has its own dual one can be defined as follows.

Definition 8 Given a first-order core fuzzy logic $L \forall$, let $L_{s, d}^{2 n} \forall$ be the expansion of $L \forall$ with new unary connectives $s_{1}, \ldots, s_{n}$ (for truth-stressers) and $d_{1}, \ldots, d_{n}$ (for truth-depressers), axioms $\left(S_{i}^{d h}\right),\left(S_{n+1}^{d h}\right),\left(D_{i}^{d h}\right),\left(S D_{i}^{d h}\right)$, and the deduction rule $\left(D R^{d h}\right)$.

$\mathrm{L}_{s, d}^{2 n} \forall$ can be obtained by expanding $\mathrm{L}_{s, d}^{2 n}$ to the first-order level.

In an analogous way to the proofs of $\mathrm{L}_{s, d}^{p, q} \forall$, we can prove the following theorems.

Theorem 6 The logic $L_{s, d}^{2 n} \forall$ is a conservative expansion of the logic $L \forall$.

Theorem 7 Let $L$ be a core fuzzy logic, $\mathbb{K}$ a class of L-chains, and $\mathbb{K}_{s, d}^{2 n}$ the class of the $L_{s, d^{-}}^{2 n}$-chains whose $s_{1}, \ldots, s_{n}, d_{1}, \ldots, d_{n}$-free reducts are in $\mathbb{K}$. Then:

(i) If $L$ has the $\mathbb{K}$ - $\sigma$-embedding property, then $L_{s, d}^{2 n} \forall$ has the $S \mathbb{K}_{s, d}^{2 n} C$.

(ii) If $L$ does not have $\mathbb{K}$-embedding property, then $L_{s, d}^{2 n} \forall$ does not have the $S \mathbb{K}_{s, d}^{2 n} C$.

In the case that $\mathbb{K}$ is the class of all standard L-chains, we have the corresponding results for the SSC of $\mathrm{L}_{s, d}^{2 n} \forall$.

\section{LOGICS WITH HEDGES BASED ON A $\Delta$-CORE FUZZY LOGIC}

In this section, we consider logics with many hedges based on $\Delta$-core fuzzy logics, where the projection $\Delta$ operator is definable.

The logic $\mathrm{L}_{s, d}^{p, q}$ based on a $\Delta$-core fuzzy logic $\mathrm{L}$ is defined as follows. 
Definition 9 Let $L$ be a $\Delta$-core fuzzy logic. A logic $L_{s, d}^{p, q}$, where $p, q$ are positive integers, is an expansion of $L$ with new unary connectives $s_{1}, \ldots, s_{p}$ (for truth-stressers) and $d_{1}, \ldots, d_{q}$ (for truth-depressers) by the following additional axioms, for $i=1, \ldots, p$ and $j=1, \ldots, q$ :

$$
\begin{array}{cl}
\left(S_{i}\right) & s_{i} \varphi \rightarrow s_{i-1} \varphi \\
\left(S_{p+1}\right) & s_{p} \overline{1} \\
\left(D_{j}\right) & d_{j-1} \varphi \rightarrow d_{j} \varphi \\
\left(D_{q+1}\right) & \neg d_{q} \overline{0}
\end{array}
$$

and the following additional deduction rule:

$\left(D R^{h}\right)$ from $(\varphi \rightarrow \psi) \vee \chi$ infer $(h \varphi \rightarrow h \psi) \vee \chi$, for each $h \in\left\{s_{1}, \ldots, s_{p}, d_{1}, \ldots, d_{q}\right\}$.

It can be proved that $\mathrm{L}_{s, d}^{p, q}$ is a semilinear logic, and in fact, it is the axiomatic expansion of $\mathrm{L}$ by the following additional axioms:

$$
\begin{array}{cl}
\left(S_{i}\right) & s_{i} \varphi \rightarrow s_{i-1} \varphi \\
\left(S_{p+1}\right) & s_{p} \overline{1} \\
\left(D_{j}\right) & d_{j-1} \varphi \rightarrow d_{j} \varphi \\
\left(D_{q+1}\right) & \neg d_{q} \overline{0} \\
\left(D R_{\Delta}^{h}\right) & \Delta(\varphi \rightarrow \psi) \rightarrow(h \varphi \rightarrow h \psi), \text { for each } h \in\left\{s_{1}, \ldots, s_{p}, d_{1}, \ldots, d_{q}\right\}
\end{array}
$$

We can obtain the first-order logic $\mathrm{L}_{s, d}^{p, q} \forall$ by expanding $\mathrm{L}_{s, d}^{p, q}$ to the first-order level in an analogous way as in Section 3, and we can have similar completeness results.

Concerning the logic $\mathrm{L}_{s, d}^{2 n}$ based on a $\Delta$-core fuzzy logic $\mathrm{L}$, we have the following definition.

Definition 10 Let $L$ be a $\Delta$-core fuzzy logic. A logic $L_{s, d}^{2 n}$, where $n$ is a positive integer, is an expansion of $L$ with new unary connectives $s_{1}, \ldots, s_{n}$ (for truth-stressers) and $d_{1}, \ldots, d_{n}$ (for truth-depressers) by the following additional axioms, for $i=1, \ldots, n$ :

$$
\begin{array}{cl}
\left(S_{i}^{d h}\right) & s_{i} \varphi \rightarrow s_{i-1} \varphi \\
\left(S_{n+1}^{d h}\right) & s_{n} \overline{1} \\
\left(D_{i}^{d h}\right) & d_{i-1} \varphi \rightarrow d_{i} \varphi \\
\left(S D_{i}^{d h}\right) & \left.d_{i} \varphi \rightarrow \neg s_{i}\right\urcorner \varphi
\end{array}
$$

and the following additional deduction rule:

$\left(D R^{d h}\right)$ from $(\varphi \rightarrow \psi) \vee \chi$ infer $(h \varphi \rightarrow h \psi) \vee \chi$, for $h \in\left\{s_{1}, \ldots, s_{n}, d_{1}, \ldots, d_{n}\right\}$.

Also, it can be proved that $\mathrm{L}_{s, d}^{2 n}$ is a semilinear logic, and in fact, it is the axiomatic expansion of $\mathrm{L}$ by the following additional axioms:

$$
\begin{array}{cl}
\left(S_{i}^{d h}\right) & s_{i} \varphi \rightarrow s_{i-1} \varphi \\
\left(S_{n+1}^{d h}\right) & s_{n} \overline{1} \\
\left(D_{i}^{d h}\right) & d_{i-1} \varphi \rightarrow d_{i} \varphi \\
\left(S D_{i}^{d h}\right) & d_{i} \varphi \rightarrow \neg s_{i} \neg \varphi \\
\left(D R_{\Delta}^{d h}\right) & \Delta(\varphi \rightarrow \psi) \rightarrow(h \varphi \rightarrow h \psi), \text { for each } h \in\left\{s_{1}, \ldots, s_{n}, d_{1}, \ldots, d_{n}\right\}
\end{array}
$$


Also, we can obtain the first-order logic $\mathrm{L}_{s, d}^{2 n} \forall$ by expanding $\mathrm{L}_{s, d}^{2 n}$ to the first-order level in a similar way as in Section 3, and we can have similar completeness results.

\section{CONCLUSION}

In this paper, we propose first-order fuzzy logics with many hedges based on a firstorder core fuzzy logic for two cases: (i) each hedge does not have a dual one, and (ii) each hedge can have its own dual one. More precisely, we expand two axiomatizations for propositional mathematical fuzzy logic with many hedges in previous works to the first-order level and prove a number of completeness results for the resulting logics w.r.t. the underlying logic. In fact, we prove that the new first-order logics are a conservative expansion of the underlying first-order core fuzzy logic and gives a characterization of their strong completeness, especially, the strong standard completeness. We also consider logics with many hedges based on $\Delta$-core fuzzy logics, which are extensions of core fuzzy logics by $\Delta$ connective. They can be obtained in an analogous way, and similar completeness results can be proved.

\section{ACKNOWLEDGEMENTS}

Funding from HUMG under grant number T16-02 is acknowledged.

\section{REFERENCES}

[1] P. Hájek, "On very true," Fuzzy Sets and Systems, vol. 124, no. 3, pp. 329-333, 2001.

[2] V. Vychodil, "Truth-depressing hedges and bl-logic," Fuzzy Sets and Systems, vol. 157, no. 15, pp. 2074-2090, 2006.

[3] F. Esteva, L. Godo, and C. Noguera, "A logical approach to fuzzy truth hedges," Inf. Sci., vol. 232, pp. 366-385, 2013.

[4] V. H. Le, F. Liu, and D. K. Tran, "Mathematical fuzzy logic with many dual hedges," in Proceedings of the Fifth Symposium on Information and Communication Technology, SoICT' 14, Hanoi, Vietnam, 2014, pp. 7-13.

[5] V. H. Le and D. K. Tran, "Linguistic logics with hedges," in Proceedings of the 2nd International Workshop on Semantic Technologies (IWOST2), Bangkok, Thailand, 2015.

[6] V. H. Le and F. Liu, "Tabulation proof procedures for fuzzy linguistic logic programming," Int. J. Approx. Reasoning, vol. 63, pp. 62-88, 2015.

[7] P. Hájek, Metamathematics of Fuzzy Logic. Dordrecht, The Netherlands: Kluwer, 1998.

[8] P. Cintula, P. Hájek, and C. Noguera, Eds., Handbook of Mathematical Fuzzy Logic, ser. Studies in Logic, Mathematical Logic and Foundations. College Publications, 2011.

[9] L. A. Zadeh, "A fuzzy-set-theoretic interpretation of linguistic hedges," Journal of Cybernetics, vol. 2, no. 3, pp. 4-34, 1972. 
[10] C. H. Nguyen and W. Wechler, "Hedge algebras: An algebraic approach to structure of sets of linguistic truth values," Fuzzy Sets and Systems, vol. 35, pp. 281-293, 1990.

[11] C. H. Nguyen and W. Wechler, "Extended hedge algebras and their application to fuzzy logic," Fuzzy Sets and Systems, vol. 52, pp. 259-281, 1992.

[12] P. Cintula, F. Esteva, J. Gispert, L. Godo, F. Montagna, and C. Noguera, "Distinguished algebraic semantics for t-norm based fuzzy logics: Methods and algebraic equivalencies," Ann. Pure Appl. Logic, vol. 160, no. 1, pp. 53-81, 2009.

\section{AUTHOR}

Van-Hung Le received a B.Eng. degree in Information Technology from Hanoi University of Science and Technology (formerly, Hanoi University of Technology) in 1995, an M.Sc. degree in Information Technology from Vietnam National University, Hanoi in 2001, and a PhD degree in Computer Science from La Trobe University, Australia in 2010. He is a lecturer in the Faculty of Information Technology at Hanoi University of Mining and Geology. His research interests include Fuzzy Logic, Logic Programming, Soft Computing, and Computational Intelligence.

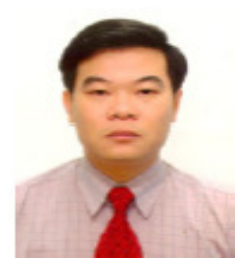

